\title{
sciendo
}

\author{
BULGARIAN ACADEMY OF SCIENCES
}

CYBERNETICS AND INFORMATION TECHNOLOGIES • Volume 20, No 2

Sofia $\bullet 2020$

Print ISSN: 1311-9702; Online ISSN: 1314-4081

DOI: $10.2478 /$ cait-2020-0017

\section{Implementation of Classic Image Transformation Algorithm to Quantum State, Boundary Extraction and Transformation of Half-Tone Image to Binary}

\author{
Sergey Gushanskiy ${ }^{1}$, Viktor Potapov ${ }^{1}$, Omar Correa Madrigal ${ }^{2}$ \\ ${ }^{1}$ Engineering Department, Southern Federal University, Taganrog, Russia \\ ${ }^{2}$ Visualization and Virtual Reality Group, Universidad de las Ciencias Informáticas, Havana, Cuba \\ E-mails:smgushanskiy@sfedu.ru_vpotapov@sfedu.ru ocorrea@uci.cu
}

\begin{abstract}
The aim of the research is computer simulation of a quantum algorithm to solve the problem of transforming a classical image using quantum computing tools and methods, studying recognition algorithms and creating a recognition model using quantum methods. The method of quantum modeling makes it possible to convert a classical image into a quantum state, select boundaries and convert a grayscale image to a binary one, and shows the possibilities of the quantum information theory in interpreting classical problems. The main results of the article are the developed quantum algorithm that allows recognizing objects, as well as the quantum method aimed at representing/processing a color pixel image. The scientific novelty of the article is expressed in the construction of a quantum system, an exponential increase in the speed of solving computational NP-complete problems, which on classical machines can be solved in unacceptable time. The motivation for writing the work was a high growth interest in quantum computing and the benefits that they guarantee. The development of the theoretical foundations of creating software systems and the design of algorithms for new information technologies and specialized computing systems is a dynamic field, as evidenced by the number of existing works in this direction. The developed algorithms for various problems of complexity classes can give a significant gain in efficiency in comparison with existing classical ones and provide a solution to a number of complex mathematical (including cryptographic) problems.
\end{abstract}

Keywords: Qubit, entanglement, quantum circuit, quantum gate, wave function, quantum algorithm.

\section{Introduction}

In modern science and technology, there is always a need to solve such strategically important tasks as weather forecast and calculation of climate changes, the creation of oncological drugs, processing signals from the Universe to search for 
extraterrestrial civilizations, processing symbolic information, cryptanalysis [1], ahead of the calculation of the trajectories of moving air and space objects and other tasks. The practical implementation of these tasks on modern, even supercomputer, systems requires an unacceptably long period of time or is generally impossible. Recently, there has been a rapid increase in interest in quantum computers [2]. Modern methods for recognizing objects have a number of significant drawbacks: search errors in large databases [3] and determining an object when changing its position, deterioration in the quality of identifying objects depending on the quality of lighting [4], masking tools [5]. It is supposed to use algorithms [6] of a quantum nature in determining objects and images. Recently, there has been a rapid increase in interest in quantum computers, especially after the sale of existing quantum computers. The use of quantum computers can significantly increase the speed of solving computational problems of higherncomplexity and, most importantly, exponentially increase the speed of solving NP complete problems that can be solved in unacceptable time on classical machines. Quantum algorithms are fairly promising field for defining objects and images. This is expressed in accelerating the computational process with the help of quantum components, stability at a different angle of the object, its motion/statics and providing cryptographic noise immunity.

\section{The process of forming a set of qubits}

A quantum computer operates with quantum bits that have two basic states $|0\rangle=\left(\begin{array}{ll}1 & 0\end{array}\right)$ and $|1\rangle=\left(\begin{array}{ll}0 & 1\end{array}\right)$. The qubit state $|0\rangle$ corresponds to the state of the electron spin in the atom "spin up", the state "spin down" corresponds to $|1\rangle$, and the mixed state (superposition of states) corresponds to the intermediate position of the spin.

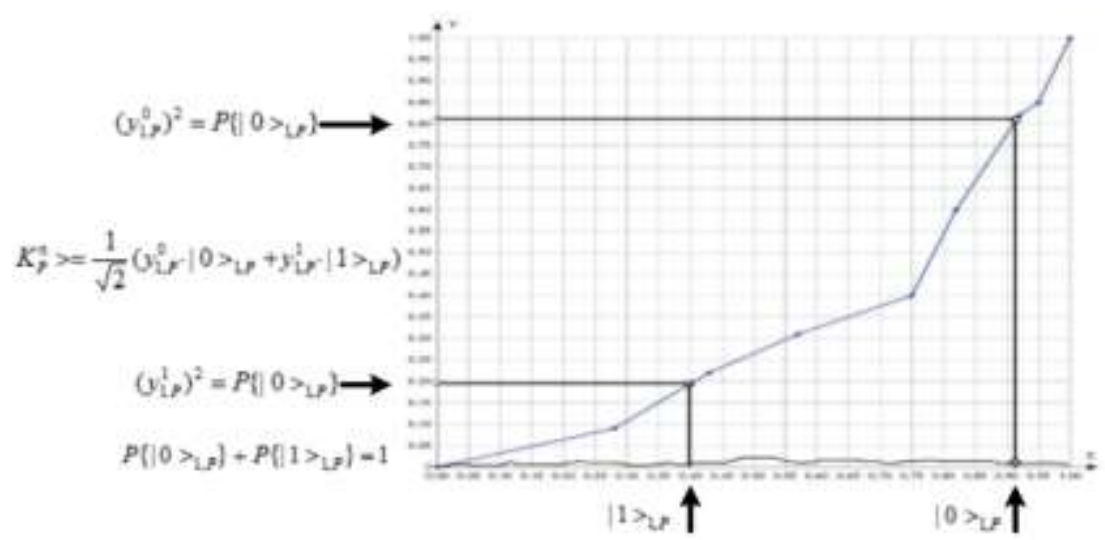

Fig. 1. The process of forming a set of qubits

Spin is the intrinsic angular momentum of elementary particles, but the spin is not related to motion in space, it is an eigenvalue of a quantum particle that cannot be explained from the standpoint of classical mechanics. In the general case, the qubit state is described by the wave function: $|\psi\rangle=\alpha_{0} \times|0\rangle+\alpha_{1} \times|1\rangle$, where $\alpha_{0}, \alpha_{1} \in C, \alpha_{0}$ and $\alpha_{1}$ are the complex amplitudes of the reading probability $|0\rangle$ or $|1\rangle$. The fact is that 
when measuring quantum bits with a standard basis with probability $\left|\alpha_{0}\right|^{2},|0\rangle$ will be obtained, and with probability $\left|\alpha_{1}\right|^{2}-|1\rangle$. In this case, the qubit will go into a state of quantum 0 or 1 . In Fig. 1 is shown the computational process when modeling entangled quantum computations in the field of quantum algorithms, which implies primarily the use of various kinds of quantum computations described in the work of quantum algorithms and the formation of a set of qubits for the state of normalization control signals at a particular moment in time.

Applying the tensor product between Hadamard transforms, we obtain terms of the form $K_{P}^{n} \otimes K_{D}^{n_{2}}$ and similar combinations of gain factors. The example described above indicates the existence of sixteen probabilistic states that describe variations among correlations according to their type and type.

\section{Converting a classic image to a state of quantum superposition}

Consider the quantum method [7], aimed at the presentation and processing of color pixel photographs or images. It is assumed that each pixel of the image $x(i, j)$ is transformed into a certain quantum state [8] $|q(i, j)\rangle:|q(i, j)\rangle=c_{0}|0\rangle+c_{1}|1\rangle$. We will encode the color palette of the pixel set in the framework of complex amplitude quantum states [9]:

$$
\delta: \mathrm{R}^{3} \rightarrow \mathrm{C}_{1}^{2},\left(\mathrm{x}_{1}, \mathrm{x}_{2} \mathrm{x}_{3}\right) \mapsto\left(\mathrm{r}_{1} \mathrm{e}^{\mathrm{i} \phi_{1}}, \mathrm{r}_{1} \mathrm{e}^{\mathrm{i} \phi_{2}}\right),
$$

where $x_{1}, x_{2}, x_{3}$ are the values of the RGB color system, $r_{1}:=\sqrt{1-x_{3}^{2}}, r_{2}:=x_{3}$, $\phi_{1}:=\arcsin \left(2 x_{1}-1\right), \quad \phi_{2}:=\arcsin \left(2 x_{2}-1\right)$. Let $\quad z_{1}=r_{1} e^{i \varphi_{1}}$, $z_{2}=r_{2} e^{i \varphi_{2}}$, then we obtain a pixel set of colors in the form $\left|q_{1}\right\rangle=z_{1}|0\rangle+z_{2}|1\rangle$. The next step is the encoding process [10] of the coordinates of the color palette of the pixel set:

(2) $|k\rangle=|x\rangle|y\rangle=\left|x_{n-1} x_{n-2} \ldots x_{0}\right\rangle\left|y_{n-1} y_{n-2} \ldots y_{0}\right\rangle, x_{i}, y_{i} \in\{0,1\}$,

where $|x\rangle,|y\rangle$ are quantum states encoding a pixel coordinate grid. Depending on the transform algorithm used, the selection of amplitude [11] probability values encoding the pixel colors of a photograph can vary significantly. Create a superposition of the computing process. Pixel superposition of states of a quantum system of a photograph or image at the input:

$$
|I\rangle=\frac{1}{2^{n}} \sum_{k=0}^{2^{2 n}-1}\left|q_{k}\right\rangle \otimes|k\rangle .
$$

The considered method of translating a classical image into a quantum state of superposition characterizes the photo image, which consists of a pixel set, in the form of a unified superposition with the characteristics of the entire set of pixels. The state vector and the probabilistic amplitude [12] are stored in the system by separate values, since we are talking about the developed model of a quantum computing device [13] and the algorithm included in the model, as well as storing the pixel set in a computing device of a classical type.

When receiving a model of a quantum computing device and a gray image algorithm and translating it into a binary state, it is necessary and sufficient to preprocess the pixel set using classical methods, tools and algorithms aimed at recognizing objects. The given process is repeated many times at several stages of 
the computational process of the algorithm. The scope of this algorithm is the task of compressing the original set of pixels by eliminating redundant information. The advantage of binary nature images is the least amount of required memory and processor time in terms of processing. The algorithm requires a number of steps:

Stage 1. Let us have an image of dimension $M \times N$ with a grayscale set of pixels. We will translate the entire set of pixels of the input photo $x(i, j)$ into the state $|q(i, j)\rangle$ of the quantum system. With the intensity of the entire pixel set, then the probability distributions $\left|c_{0}\right|^{2}$ and $\left|c_{1}\right|^{2}$ are calculated by determining the sums $s_{1}$ and $S_{2}$ as follows:

$$
s_{1}=\sum_{k=-1}^{1} \sum_{l=0}^{1} x(i-k, j-l)+x(i, j+l)+x(i, j+2),
$$

$$
s_{2}=x(i-1, j-1)+x(i-1, j)+x(i, j-l) \text {. }
$$

If $P=\left(s_{1}+s_{2}\right) / 5, \quad$ then $\left|c_{0}\right|^{2}=f(P), \quad\left|c_{1}\right|^{2}=1-f(P), \quad$ where $f(P)=\frac{1}{1+e^{\frac{P+a}{b}}}$. The quantum states $|0\rangle,|1\rangle$ correspond to the vectors $\left(\begin{array}{l}1 \\ 0\end{array}\right),\left(\begin{array}{l}0 \\ 1\end{array}\right)$. In accordance with this qubit $|q(i, j)\rangle$, the vector $\left(\begin{array}{c}1-f(P) \\ f(P)\end{array}\right)$ is mapped, so the intensity of the pixel set of the photograph is represented in two-dimensional space.

Stage 2. By measuring the qubit $|q(i, j)\rangle$ of the entire pixel set of the photo fed to the input, the matrix of the pixel system is formed [14]. If the quantum states $|0\rangle,|1\rangle$ correspond to the indicators 0,1 of the output pixel set of the photo, then we get a binary object at the output. Fig. 2 demonstrates the model being developed of a quantum computing device and the result of a quantum algorithm for converting a set of pixels. This software development is an offline desktop computing system with an open architecture, but in the future, it is planned to broadcast it online.

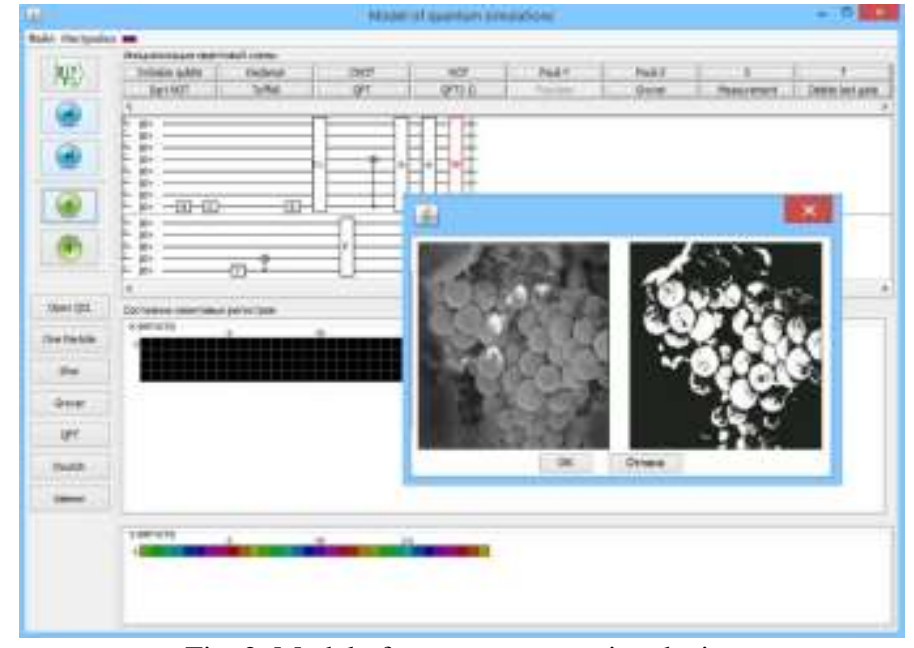

Fig. 2. Model of quantum computing device

The measurement process consists in playing out a random number from the interval $[0,1]$. When a value falls into the interval $\left[0,\left|c_{1}\right|^{2}\right]$, the output value of the measuring process is the basis state of the sconces of vector 0 , otherwise (the interval $\left.\left[\left|c_{1}\right|^{2}, 1\right]\right)$ is the quantum state of sconces of vector 1 . The developed algorithm uses 
quantum principles, such as a superposition of quantum states of a computing system, entanglement of quantum states, and the transformation of a classical image into a quantum state by encoding a color palette of a pixel set within complex amplitude quantum states.

\section{Implementation of quantum support vector algorithm}

In the latest quantum computer technology, it is possible to control and control only a limited number of qubits. Whereas, on the other hand, the real problem of optical character recognition in the quantum support vector method requires dozens of qubits, which cannot be implemented at present. Thus, we restrict the problem to the case with minimal costs, in which only two options ("6" or "9") are in the list and only two properties (horizontal or vertical position, which will be determined later) make up the problem. This allows us to demonstrate this algorithm of quantum artificial intelligence based on the 4-qubit moment of the core of a quantum processor at room temperature. The quantum support vector method here works as follows: first, the machine is trained in two printed images with the standard font of options, as shown in Fig. 3, and then handwritten images of options "6" or "9" are provided (as in Fig. 4), and the machine recognizes (classifies) each of them in order to determine which species group it belongs to.

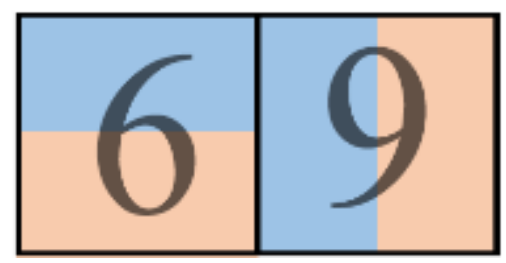

Fig. 3. Standard Times New Roman font for two characters "6" and "9" for OCR preparation

The vertical (horizontal) position is calculated in pixels from the left (upper) half to the right (lower) (Fig. 3). It is worth noting that, in fact, the machine works with a vector formed by the properties of each image, and before sending to the machine, each image must undergo pre-processing, such as, for example, resizing pixels.

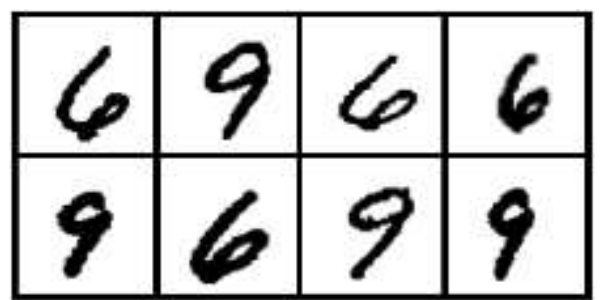

Fig. 4. Handwritten test samples for support vector algorithm

In addition, we applied linear transformation of vector properties in order to ensure compatibility with the machine. After this pre-processing, two printed images 
with standard fonts can be represented as $x_{1} \overrightarrow{ }=(0.9872,0.1595)$ for option " 6 " and $x_{2} \overrightarrow{ }=(0.3544,0.9351)$ for option " 9 ".

Let's move on to the experimental implementation of the quantum optical character recognition algorithm. To implement a 4-qubit quantum processor, we take the widely used nuclear magnetic resonance. The experiments are carried out on a Bruker AV-400 306K spectrometer. The sample used is cyodotrifluoroethylene $\left(\mathrm{C}_{2} \mathrm{~F}_{3} \mathrm{I}\right)$ dissolved in $d$-chloroform, which consists of ${ }^{13} \mathrm{C}$ nuclear spin (physical terms) and three 19F nuclear spins. The natural Hamiltonian (Hamiltonian function) of this loosely coupled system of spins in a rotating reference frame is

$$
H_{\mathrm{NMR}}=\sum_{j=1}^{4} \pi v_{j} \sigma_{z}^{j}+\sum_{1 \leq j<k \leq 4} \frac{\pi}{2} J_{j k} \sigma_{z}^{j} \sigma_{z}^{k},
$$

Deviation of the density matrix of the thermal equilibrium state: $\rho_{\text {eq }}=\sum_{i=1}^{4} \gamma_{i} \sigma_{z}^{i}$, where $\gamma_{i}$ represents the gyromagnetic position of each nuclear spin. The experimental procedure consists of three parts: 1) pseudo-pure state of preparation; 2) the construction of the kernel of the matrix $K$; and 3) machine learning and classification. Starting with the thermal state $\rho_{\text {eq }}$, the linear sampling method is used for preparation (PPS): $\rho_{i}=\frac{1-\varepsilon}{16} I_{16}+\varepsilon|0000\rangle\langle 0000|$. Here $I_{16}$ is a $16 \times 16$ identification operator, and $\varepsilon \approx 10^{-5}$ is the polarization. In the quantum scheme of the second part, the first qubit $\left({ }^{13} \mathrm{C}\right)$ serves as a test qubit, and the second qubit (training set of registers) is encoded with the information of two standard samples of options " 6 " and " 9 ". The source training data is implemented using two controlled interlaces. After that, the structure of the test qubit will represent the core of the matrix $K$ up to a constant factor $\operatorname{tr} K$. In the experiment, the density matrix $K / \operatorname{tr} K$ is measured as $\left(\begin{array}{ll}0.5065 & 0.2425 \\ 0.2425 & 0.4935\end{array}\right)$ using the quantum structural tomography technique.

The third part of the experiment is classification. The training set is encoded in the system register (third qubit). In this case, we carry out the reduction without bias, i.e., $b=0$. Then the linear Equation (1) reduces to $F \tilde{a} \equiv\left(K+\gamma^{-1} I_{2}\right) \vec{a}=\vec{y}$ of dimension $2 \times 2$. The weight set by the user is set as $\gamma=2$. By rotation around the $y$ axis, i.e., $R_{y}^{-\pi / 4}$, the system register is converted to the form $|y\rangle=\left(\begin{array}{c}1 \\ -1\end{array}\right) / \sqrt{2}$. Then, a phase estimation algorithm is used to implement the inversion of the matrix, which generates the state $\mathrm{F}^{-1}|\mathrm{y}\rangle$. At this stage, the weight of the support vectors is calculated and loaded into the system register when the next qubit is in the state $|1\rangle$. By recalling the original training data, the weights are encoded with the coefficient of the reference vectors $\left|x_{j}\right\rangle$ related to them, leading to the preparation of the training data state $|\tilde{u}\rangle$. Then the inverse operation $U_{x 0}$ is applied, which refers to the request state $x_{0}$. After inversion of the matrix $F^{-1}$, these operations must be caused by rotation. In the experiment, the matrix inversion circuit is placed in the same pulse, optimized by the pulse enhancement gradient by the technical method (GRAPE), with pulse duration of $20 \mathrm{~ms}$ and the number of segments 1000. The rest of the circuit is placed in another GRAPE pulse with a duration of $25 \mathrm{~ms}$ and 2000 segments. All pulses theoretically have an accuracy of more than $99 \%$ and are designed for reliability compared to nonuniform radio frequency pulses. Finally, the classification result is read through the 
mean value of the coherent expression of the auxiliary qubit $|000\rangle\langle 000|\otimes| 1\rangle_{A}\left\langle\left. 0\right|_{A}\right.$. In the experiment, the information of the auxiliary qubit is transmitted to the ${ }^{13} \mathrm{C}$ back using a swap (the operation of exchanging memory pages with external RAM), and then it is read through the ${ }^{13} \mathrm{C}$ spectrum. If the corresponding peak in the ${ }^{13} \mathrm{C}$ spectrum is directed upwards (example of Fig. 5), the classification result is positive, and therefore, the recognized symbol is " 6 ". Conversely, when the peak is directed downward, the classification result will be "9". The experiment presents 8 different recognition problems corresponding to 8 handwritten characters in Fig. 5, which shows the recognition results.

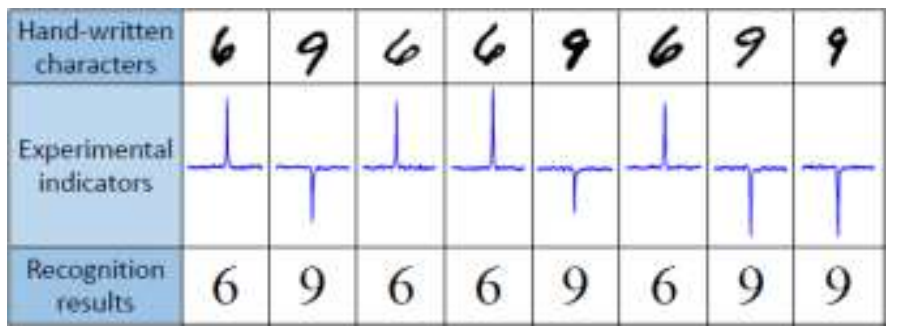

Fig. 5. Handwritten character recognition result

In conclusion, we demonstrate the first artificial intelligence algorithm implemented quantum-mechanically on a 4-qubit quantum processor, i.e., a quantum learning machine. As an example, a quantum vector reference machine was used to solve the problem of optical character recognition. In an experiment, a quantum machine learns the standard character fonts " 6 " and " 9 ," and then is used to classify incoming handwritten characters. Successful classification shows the ability of a machine to learn and work as an intelligent person. This quantum machine can be expanded for a large-scale data set, using resources only logarithmically in the dimension of objects and the amount of training data.

\section{Conclusion}

The main and fundamental benefits of using quantum computing in various fields of knowledge are: operators.

1. The potential for accelerating computations using quantum evolutionary

2. The introduction of quantum superposition and correlation operators into the classical algorithm, as well as the probabilistic nature of the quantum algorithm, entail the appearance of unique properties of the data processing process, which affects the result of the algorithm.

3. Weak dependence on environmental interference, independence from the static or motion of an object or camera.

4. Transmission of a full-fledged video image through low-speed communication channels.

5. Providing a high level of security when trying to interfere with image control channels. 
The desire to increase the computing power of computers and provide an unsurpassed scale of tasks is one of the determining factors in the development of supercomputer technologies. The main advantages of using quantum computing [15] in the field of determining objects and images: acceleration of the computing process using quantum components, stability at different angles of the object, its motion / statics, ensuring cryptographic noise immunity. Great importance is attached to the development of fundamentally new physical principles of computing, where quantum computing is the most promising area. The disadvantages of modern methods of image recognition: the error when searching through large databases, the recognition error when changing the angle of the object, the effect of lighting on the quality of recognition, age-related changes, masking signs, the sensitivity of the software to facial expressions. In this paper, it is proposed to use modern quantum algorithms for pattern recognition. Using the basis of the presented quantum algorithms in combination with the existing classical algorithms, would make it is possible to realize effective face recognition, which will overcome (in whole or in part) many of these shortcomings.

Acknowledgments: The reported study was funded by RFBR according to the research project No 19-07-01082.

\section{References}

1. G a o, C., D. Ji an g, Y. Gu o, L. Ch e n. Multi-Matrix Error Estimation and Reconciliation for Quantum Key Distribution. - Opt. Express, Vol. 27, 2019.

2. Kiktenko, E., A. Malyshev, A. Bozhedarov, N. Pozhar, M. Anufriev, A. Fe d o r o v. Error Estimation at the Information Reconciliation Stage of Quantum Key Distribution. - J. Russ. Laser Res., Vol. 39, 2018.

3. Potapov, V., S. Gushansky, V. Guzik, M. Polenov. Architecture and Software Implementation of a Quantum Computer Model. - In: Advances in Intelligent Systems and Computing. Springer Verlag. Vol. 465. 2016, pp. 59-68.

4. B i a monte, J., P. Wittek, N. Pancotti, P. Rebentrost, N. Wiebe, S. Lloyd. Quantum Machine Learning. - Nature, Vol. 549, 2017, No 7671.

5. D a s, S., G. S i o p s is, C. We edbrook. Continuous-Variable Quantum Gaussian Process Regression and Quantum Singular Value Decomposition of Nonsparse Low-Rank Matrices. Physical Review A, Vol. 97, 2018, No 2.

6. T a n g, E. A Quantum-Inspired Classical Algorithm for Recommendation Systems. - In: Proc. of 51st Annual ACM SIGACT Symposium on Theory of Computing, ACM, 2019, pp. 217-228.

7. Abhijith, J., A. Patel. Spatial Search on Graphs with Multiple Targets Using Flip-Flop Quantum Walk. - Quantum Information and Computation, Vol. 18, 2018, pp. 1295-1331.

8. B r a nd ão, F. G. S. L., M. J. K a s to ry a no. Finite Correlation Length Implies Efficient Preparation of Quantum Thermal States. - Commun. Math. Phys., Vol. 365, 2019, No 1, arXiv:1609.07877.

9. Potapov, V., S. Gushanski y, M. Polenov. The Methodology of Implementation and Simulation of Quantum Algorithms and Processes. - In: Proc. of 11th IEEE International Conference on Application of Information and Communication Technologies (AICT'17), Institute of Electrical and Electronics Engineers, Moscow, 20-22 September, 2017, pp. 437-441.

10. Motta, M., C. S u n, A. T. K. T a n, M. J. O'rourke, E. Ye, A. J. M inni ch, F. G. S. L. $\mathrm{B}$ r a n d ã o, G. K i n-Li c C h a n. Quantum Imaginary Time Evolution, Quantum Lanczos, and Quantum Thermal Averaging. - 2019, arXiv:1901.07653v1. 
11. S c h midt, L., S. S a n t u r k a r, D. T s i pras, K. T a l w a r, A. M a d r y. Adversarially Robust Generalization Requires More Data. - In: Advances in Neural Information Processing Systems, 2018, pp. 5019-5031.

12. C a s a r e s, P., M. M a r t i n-D e $1 \mathrm{~g}$ a d o. A Quantum in Predictor-Corrector Algorithm for Linear Programming. - arXiv preprint arXiv:1902.06749, 2019.

13. A a ro n s o n, S., D. Grier, L. S ch a effer. A Quantum Query Complexity Trichotomy for Regular Languages. - Electronic Colloquium on Computational Complexity (ECCC), Vol. 26, 2018, No 61.

14. Srinivasan, S., C. Downey, B. B oots. Learning and Inference in Hilbert space with Quantum Graphical Models. - In: Advances in Neural Information Processing Systems, Vol. 31, 2018.

Received: 08.01.2020; Second Version: 23.04.2020; Accepted: 14.05.2020 (fast track) 